\title{
Novo método para determinação da temperatura de corpos imersos em plasma
}

New method for determination of temperature of body immersed in plasma

Erisson Aparecido de Deus Leal ${ }^{1}$, Sandi Itamar Schafer de Souza' ${ }^{1}$, Clodomiro Alves Junior ${ }^{2}$

\section{RESUMO}

Quando um sólido é imerso em plasma, dois tipos diferentes de transferência de energia ocorrem: por radiação e por colisão de partículas. No primeiro caso, a energia é transferida de maneira uniforme e paralela à superfície. No segundo caso, ela é transferida para as vizinhanças das micro-regiões onde os picos térmicos são gerados. A fim de estudar esses dois efeitos, discos paralelos às amostras, fabricados de aço AISI M35, cujos valores de dureza são fortemente sensíveis à temperatura de revenimento, foram utilizadas como micro sensores térmicos. Através da correlação dos valores de dureza com a temperatura, foi possível obter o perfil térmico dos discos de aço AISI M35 tratadas por plasma. Verificouse que discos paralelos revenidos em plasma apresentaram camadas nas duas superfícies (interna e externa) do confinamento, que não se encontraram presentes nos discos revenidos em forno resistivo. Além disso, verificou-se que os discos que apresentaram maior gradiente térmico quando revenidas em plasma, foram as tratadas na configuração cátodo oco com pressão de 2 mbar com termopar a $550^{\circ} \mathrm{C}$ como referência.

Palavras-chave: Gradiente térmico; Cátodo oco; Aço rápido AISI M35; Plasma.

\begin{abstract}
When a solid is immersed in plasma, two different types of energy transfer occurs: by radiation and collision of particles. In the first case, the energy is transferred evenly and parallel to the surface.In the latter case, it is transferred to the neighborhood of micro areas where thermal spikes are generated. In order to study these two effects, discs parallel to the samples made of AISI M35, whose hardness values are strongly temperature sensitive, were used as micro thermal sensors. By correlating hardness to temperature, it was possible to obtain the thermal profile of AISI M35 steel discs treated by plasma. It has been found that parallel disks with layers quenched by plasma had both surfaces (internal and external) of the containment that were not present in the furnace tempering resistive discs. Furthermore, it was found that discs with higher thermal gradients in plasma when annealed, were treated in the hollow cathode configuration with a pressure of $2 \mathrm{mbar}$ and thermocouple at $550^{\circ} \mathrm{C}$ as the reference.
\end{abstract}

Keywords: Thermal gradients; Hollow cathode; AISI M35 steel; Plasma.

\footnotetext{
'Universidade Federal do Rio Grande do Norte (UFRN) - Natal (RN), Brasil. ¿Universidade Federal Rural do Semiárido (UFERSA) - Mossoró (RN), Brasil. Endereço para correspondência: Erisson Aparecido de Deus Leal - Av. Salgado Filho, 3000 - CEP: 59072-970 - Natal (RN), Brasil. E-mail: erisson_eng_mec@hotmail.com

Recebido em: 25/06/2014 Aprovado em: 26/11/2014
} 


\section{INTRODUÇÃO}

Dentre as novas fontes energéticas para processamento de materiais, o plasma tem se destacado como uma alternativa para a indústria devida a sua versatilidade. Em certas aplicações como na siderurgia (fundição, redução de óxidos), metalurgia (tratamentos termoquímicos, sinterização, solda e corte) ou até mesmo no processamento de resíduos perigosos (resíduos organometálicos, lamas de eletrodeposição, borras de produção de alumínio, cinzas de incineração e lixo hospitalar), a compreensão da forma de aquecimento por plasma e seus efeitos sobre a microestrutura e propriedades dos materiais assim tratados tornam-se cruciais para o desenvolvimento de materiais por essa nova tecnologia ${ }^{1}$.

Entretanto, existem poucos modelos matemáticos e/ou experimentais que simulem uma situação real de aquecimento de sólidos quando imersos no mesmo. Sabe-se que no plasma, ao contrário dos processos convencionais, a transferência de energia ocorre por radiação do plasma para a superfície do sólido, bem como a partir dos picos térmicos criados na superfície devido ao bombardeamento das partículas ${ }^{2}$.

Dessa forma, espera-se que os materiais tratados termicamente por plasma apresentam um comportamento diferente de outros materiais tratado por formas convencionais de aquecimento. Sabe-se da literatura que a região aquecida por plasma produz uma modificação na microestrutura do material, promovendo alterações na sua dureza e resistência mecânica, uma vez que o plasma é capaz de produzir elevadas taxas de aquecimento nas peças tratadas ${ }^{3}$.

Foi observado que um gradiente de porosidade foi obtido em amostras compactas de pós microligados de bronze de alumínio, a partir da sua superfície para região interior ${ }^{4}$. A explicação dada para esse fenômeno foi a fusão local de partícula do pó, produzida pelos picos térmicos gerados, e a consequente percolação nos poros internos, aumentando assim a porosidade na superfície. A literatura mostra que, dependendo da configuração do plasma, o efeito de gradiente térmico pode ser mais acentuado ${ }^{5}$. Quando a característica geométrica de uma descarga luminescente convencional (configuração cátodo planar) é modificada para configuração de cátodo oco, a taxa de ionização é aumentada em mais de 100 vezes, gerando um plasma com alta taxa de colisão ${ }^{6}$. As amostras tratadas usando esta configuração dependem da distancia entre cátodos, pressão do gás e voltagem ${ }^{4}$. Embora existam evidências sobre o gradiente térmico gerado em sólidos imersos em plasma, pouco se sabe quantitativamente esses valores. A medida da temperatura pontual sendo o maior obstáculo para essa quantificação.

Sabe-se que alguns aços temperados possuem valores de dureza altamente sensíveis à temperatura de revenimento. Estes aços, quando temperados e revenidos no forno resistivo, apresentam um comportamento sigmoidal na propriedade de dureza em função da temperatura de revenimento. Isso ocorre porque os carbetos finamente dispersos existentes na estrutura temperada passam por um processo de coalescimento durante o revenimento. Dentre estes aços, pode-se citar o aço rápido AISI M35.

Este trabalho tem por objetivo propor uma metodologia para determinar a temperatura, de forma indireta, em diferentes micro-regiões, de discos de aço AISI M35 tratadas termicamente por plasma e, com isso, quantificar o perfil de temperatura e avaliar gradientes térmicos da superfície para seu interior. Para isso utilizar-se-á como micro sensor térmico (sonda), discos de aço AISI M35.

\section{METODOLOGIA EXPERIMENTAL}

Discos fabricados de aço AISI M35 ( $\mathrm{D}=33,45 \mathrm{~mm}$ e $\mathrm{H}=1 \mathrm{~mm}$ ) foram inicialmente aquecidos até temperatura de $1230^{\circ} \mathrm{C}$, seguido de resfriamento rápido (têmpera). Em seguida, parte desses discos foram revenidas em forno resistivo às temperaturas de 550 , $580,610,640,700$ e $750^{\circ} \mathrm{C}$ para obtenção da curva de calibração da dureza versus temperatura de revenimento. Os resultados obtidos nessa curva foram utilizados para determinação do perfil de temperatura das amostras imersas em plasma, a partir do perfil de dureza. A outra parte dos discos, que não foi revenida em forno resistivo, foram colocados para revenimento em plasma. Eles foram colocados no equipamento de plasma, conforme ilustrado na Fig. 1. Para diferenciá-los foi chamado de amostra aquele colocado diretamente sobre o porta- amostra e de disco paralelo o outro equilibrado nas hastes metálicas conforme detalhado na Fig. 1b). Os discos imersos em plasma foram também previamente temperados nas mesmas condições anteriores.

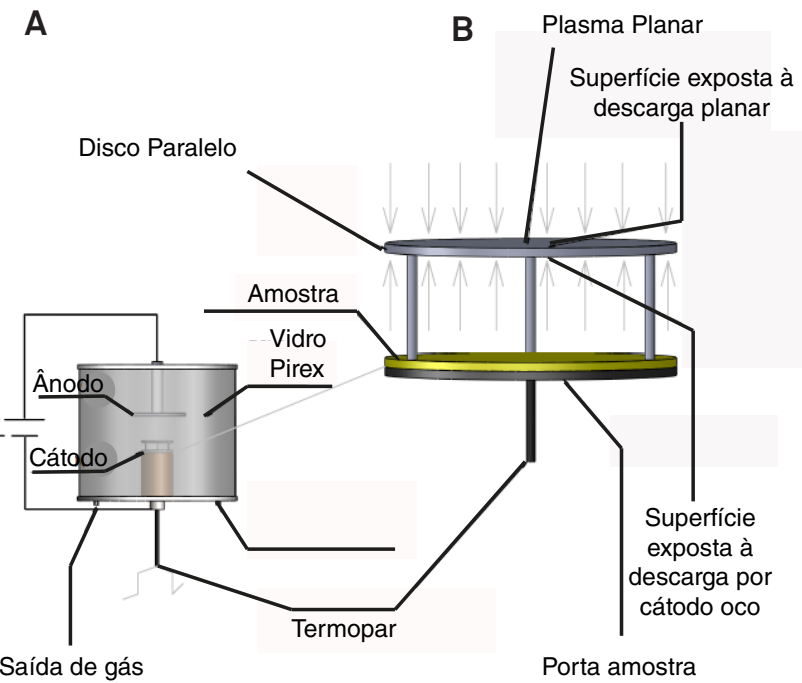

Figura 1: (A) Modelo esquemático do equipamento de plasma utilizado para revenimento das amostras temperadas de aço AISI M35. (B) Configuração cátodo oco.

O reator cilíndrico de borossilicato possui altura de $300 \mathrm{~mm}$ e diâmetro de $180 \mathrm{~mm}$. Ele é fechado por dois flanges de aço inoxidável, vedados por gaxetas de nitrila com perfil em L. A fonte de tensão de saída DC é ajustada através de um Varivolt tipo VM 215. 
A fonte de tensão usada tem a seguinte especificação: tensão de entrada, $220 \mathrm{~V}$; tensão máxima de saída sem carga, $1200 \mathrm{~V}$; corrente máxima de entrada, 5,5 A; corrente máxima de saída, $1 \mathrm{~A}$.

No flange superior está a conexão do anodo, sensor de pressão e dispositivo para quebra de vácuo. No flange inferior está inserido o cátodo, termopar e a entrada e saída de gases. O cátodo é composto de um tubo de aço inoxidável, por onde passa o termopar, em cuja extremidade tem-se uma plataforma. O conjunto é revestido por um material cerâmico. Observando a Fig. 1b) tem-se que duas configurações de descarga são formadas: Descarga planar e descarga em cátodo oco. Para a configuração planar é colocada apenas uma mesa de aço inoxidável, com diâmetro de 33,45 mm que servirá como porta amostra. Para a configuração cátodo oco é adicionado um disco paralelo ao porta amostra, com mesmo diâmetro, apoiado por um tripé de fios de 1,2 $\mathrm{mm}$ de espessura, formando então um cátodo oco com distância entre cátodo de 12 $\mathrm{mm}$. Nessa configuração a amostra é colocada entre os dois cátodos. O uso deste disco paralelo (configuração catodo oco), aumenta a densidade de íons, tornando mais efetivo a transferência de energia por colisão.

Para revenir os discos em plasma considerou-se a temperatura do termopar fixado no porta amostra como referência. Utilizouse para revenimento a plasma, a configuração em cátodo oco e foi utilizada a pressão de 2 mbar e, também a de 4 mbar com a mesma temperatura de referência $\left(550^{\circ} \mathrm{C}\right)$ em atmosfera de argônio. Para o outro revenimento em plasma, foi utilizada a mesma configuração, ou seja, cátodo oco e a pressão utilizada foi somente a de 2 mbar em atmosfera de argônio com a temperatura de referência no termopar $\left(400^{\circ} \mathrm{C}\right)$. O tempo de revenimento em todas as condições teve duração de $2 \mathrm{~h}$.

Após o revenimento em plasma e em forno resistivo, ensaios de microdurezas foram realizados na secção transversal das amostras. As impressões foram produzidas nas amostras utilizando carga de 10 gf, de forma equidistante uma da outra com espaçamento de 0,02 $\mathrm{mm}$ na vertical e na horizontal, da borda para o centro da amostra, conforme ilustrado na Fig. 2.

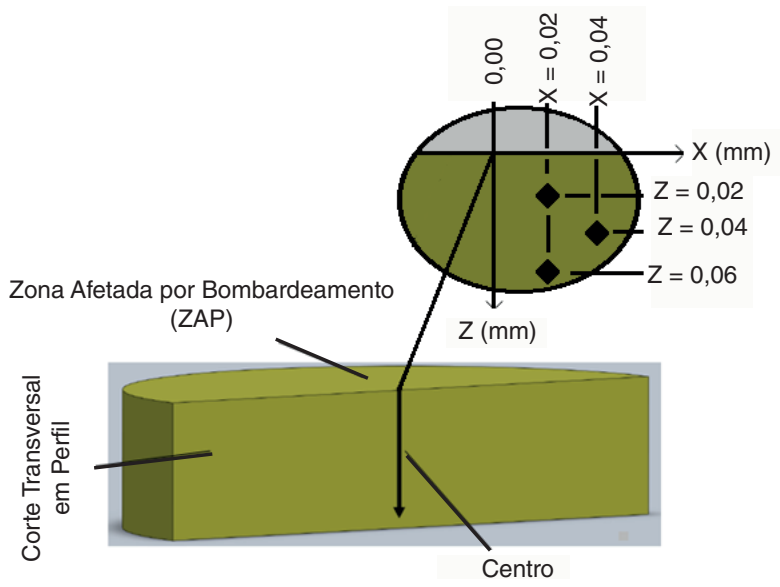

Figura 2: Representação do corte da amostra em perfil, indicando a região e a direção, em que os ensaios de microdureza foram realizadas.
A partir dos valores de dureza medidos nas amostras revenidas em forno resistivo, estes valores nos pontos avaliados, não variaram ao longo da seção transversal para cada tratamento. Os tratamentos de revenimento em forno resistivo foram realizados para as temperaturas de $550,580,610,640,700$ e $750^{\circ} \mathrm{C}$. Isso possibilitou a construção do gráfico, dureza versus temperatura de revenimento mostrado na Fig. 3. A partir do comportamento exposto no gráfico montou-se a equação aproximada para descrever a variação da temperatura em função da dureza. Esta equação foi usada como referência, para inferir a temperatura no processo de revenimento a plasma, ou seja, para determinar, de maneira indireta, os valores da temperatura para construção do perfil térmico das amostras revenidas em plasma.

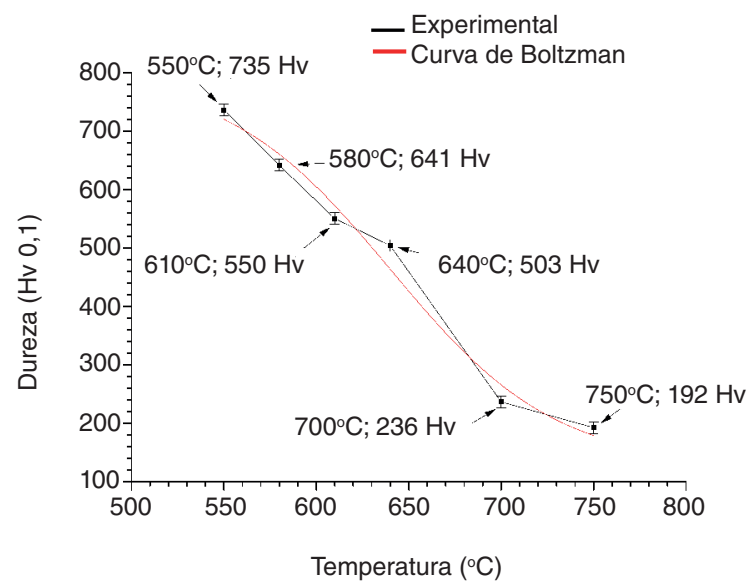

Figura 3: Curva dureza versus temperatura de revenimento para amostras tratadas em forno resistivo.

Um ajuste da curva foi realizado através de uma linha de tendência sigmoidal, baseada no modelo de Boltzmann, dado pela equação:

$$
T\left({ }^{\circ} \mathrm{C}\right)=\operatorname{Ln}\left(\frac{(A 1-A 2)}{D(H v)-A 2}-1\right) d_{x}+x_{0}
$$

onde:

$\mathrm{T}\left({ }^{\circ} \mathrm{C}\right)$ é a temperatura revenida; $\mathrm{D}(\mathrm{H} v)$ é o valor da dureza aplicada; $\mathrm{d}_{\mathrm{x}}$ é a largura da faixa ou nível de espalhamento onde ocorreram as maiores variações de dureza; A1 representa a assíntota inferior, A2 é a assíntota superior; $\mathrm{x} 0$ é o ponto de inflexão do gráfico.

A Eq. 1 originária do ajuste da curva dureza versus temperatura de revenimento no forno resistivo, foi usada para determinar indiretamente o perfil térmico das amostras revenidas em plasma.

\section{RESULTADOS E DISCUSSÕES}

O comportamento do perfil de dureza com a profundidade, dos discos paralelos às amostras revenidas em plasma, foi estudado e percebeu-se que, diferente da uniformidade encontrada nas amostras revenidas em forno resistivo, houve uma variação 
significativa do valor da dureza ao longo da sua profundidade. De um modo geral, esses discos imersos em plasma apresentaram gradientes de dureza nas duas superfícies, menores que no seu interior. Na superfície exposta à descarga por cátodo oco (confinado), os picos foram gerados por plasma com alta densidade de íons. No lado externo, a superfície foi exposta à descarga planar. A Fig. 4 ilustra esse comportamento para revenimento realizado na temperatura de referência de $550^{\circ} \mathrm{C}$.
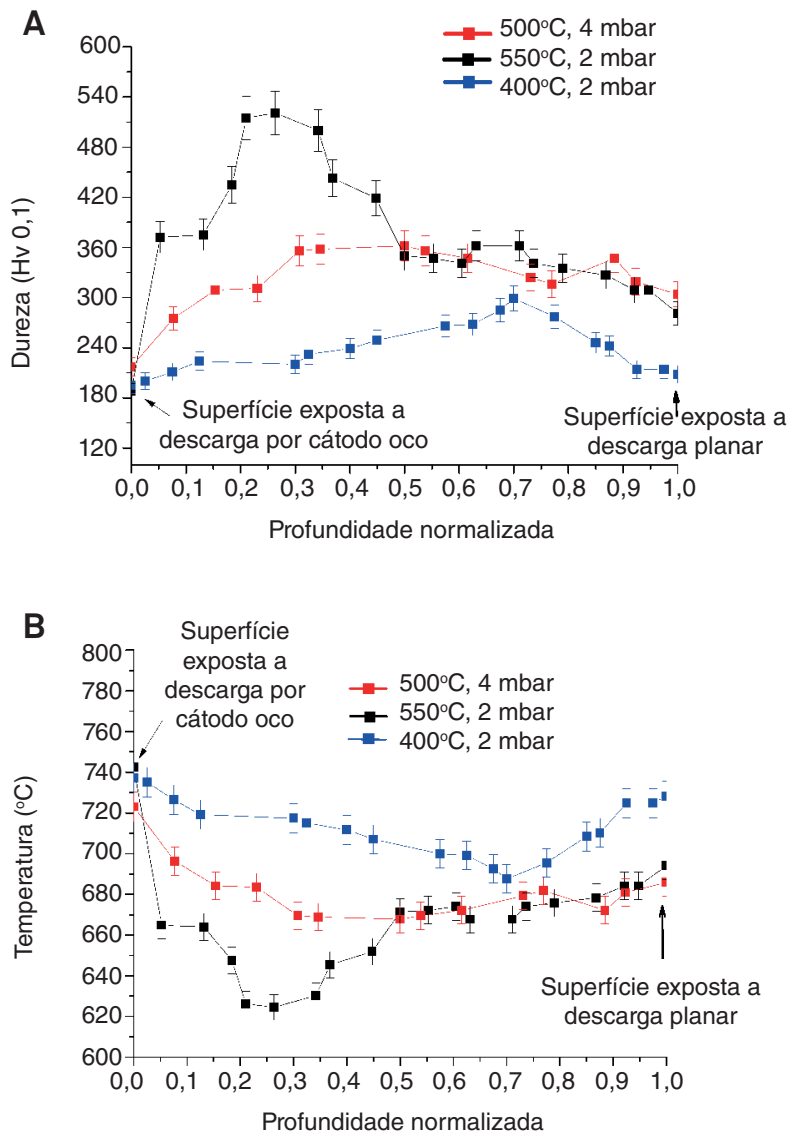

Figura 4: Comportamento de discos revenidos em plasma tendo uma das faces exposta à descarga por cátodo oco e a outra face a uma descarga planar. (A) Perfil de dureza; (B) Perfil de temperatura obtida a partir da curva $(A)$.

Como podem ser vistos nos resultados, a dureza aumenta da superfície para o interior do material e depois decresce até o lado oposto para todas as condições estudadas. Para revenimentos realizados na pressão de 2 mbar, as diferenças de dureza entre a superfície exposta à descarga por cátodo oco e o centro da amostra foram de $300 \mathrm{Hv}$ e $105 \mathrm{Hv}$, respectivmente, para a temperatura de referência de $550^{\circ} \mathrm{C}$ e $400^{\circ} \mathrm{C}$. Para amostras revenidas na pressão de 4 mbar e temperatura de referência de $550^{\circ} \mathrm{C}$, a diferença de dureza foi de $150 \mathrm{Hv}$. Além disso, pode-se também verificar diferenças na dureza entre as superfícies expostas à descarga por cátodo oco e aquelas expostas às descargas planares.

Esses resultados, de acordo com o gráfico da Fig. 3, implicam em diferenças de temperatura como ilustradas na Fig. 4b. Eles indicam que houve uma variação de temperatura entre a região superficial e o centro dos discos. Estes fenômenos podem ser explicados devido ao efeito do bombardeamento iônico na superfície dos materiais tratados em plasma. Sabe-se que a transferência térmica do plasma para a superfície se faz principalmente pela radiação e pelo bombardeamento de íons e espécies neutras energéticas ${ }^{7}$. No processo de bombardeamento, em cada colisão ocorre um pico térmico, sendo depois difundido para vizinhança ${ }^{2}$. Dependendo da taxa de colisão e da condutividade térmica dos materiais, as características da camada poderão variar.

Para superfícies tratadas por descarga em cátodo oco, devido ao confinamento do plasma, a taxa de ionização será maior, resultando em maior taxa de bombardeamento. Para menores pressões, a energia por íon será maior, implicando em maiores picos térmicos e, conseqüentemente, maior gradiente térmico.

As curvas da Fig. 4b mostram o perfil térmico desde a superfície exposta à descarga por cátodo oco (origem) até a superfície exposta à descarga planar. Diferença de temperatura tão alta quanto $120^{\circ} \mathrm{C}$ foi observada entre a superfície exposta à descarga por cátodo oco e o núcleo do disco para o revenimento realizado a 2 mbar e temperatura de referência de $550^{\circ} \mathrm{C}$. Para a superfície exposta à descarga planar essa diferença foi de apenas $70^{\circ} \mathrm{C}$. Ou seja, a região bombardeada e irradiada pelo plasma na configuração cátodo oco (configuração com alta densidade de íons) atingiu temperatura maior que no interior do disco. O outro lado do disco, ou seja, superfície exposta ao plasma em configuração planar (baixa densidade de íons), a temperatura ficou mais alta que a do interior do disco porque essa superfície é irradiada e bombardeada por espécies do plasma planar. Como essa configuração possui uma densidade de íons inferior que a primeira, a temperatura nesta superfície é inferior àquela exposta ao plasma na configuração cátodo oco.

\section{Análise Quantitativa}

Uma análise quantitativa aproximada foi realizada para determinar a energia transferida aos discos paralelos, $\Delta$ Ear, através das curvas dos resultados obtidos dos perfis térmicos das amostras tratadas termicamente por plasma, conforme Figs. 4 e 5, dadas pela seguinte equação ${ }^{2}$ :

$$
\Delta E_{a r}=\int m c_{p} d(\Delta T)
$$

O termo $\Delta \mathrm{T}$ representa a diferença entre a temperatura na posição analisada e a temperatura ambiente, assumida Tamb $=25^{\circ} \mathrm{C}$. O material de trabalho utilizado foi o aço rápido AISI M35. A Eq. 2 pode ser reescrita da seguinte forma:

$$
\Delta E_{a r}=\rho_{a c o} \pi \frac{D^{2}}{4} c_{p_{a c o}} \int z d(\Delta T)
$$

Aproximando o aço rápido AISI M35 para o aço inoxidável AISI 302 a $300 \mathrm{~K}$, tem-se que $\rho \mathrm{a}_{\text {ço }}=8055 \mathrm{~kg} / \mathrm{m} 3$ e $c_{\text {paço }}=480 \mathrm{~J} / \mathrm{kgK}^{8}$. Substituindo na equação da energia acima, onde o diâmetro é: 
$\mathrm{D}=33,45 \mathrm{~mm}$, obtém-se a contribuição da quantidade de energia transferida para os discos paralelos, nas suas devidas configurações de trabalho.

Portanto, substituindo todos os dados fornecidos através da Eq. 3 e aplicando numericamente a integral pelo método de Riemann ${ }^{9}$ para a curva da profundidade $\mathrm{z}$ com a variação de temperatura $\Delta \mathrm{T}$, conforme Figs. 4 e 5 para todas as situações avaliadas, obteve-se a análise quantitativa para os tratamentos conforme Tabela 1.

Os tempos dos tratamentos foram de 2 horas, mas o regime permanente foi registrado nos períodos de $75 \mathrm{~min}$ para o disco paralelo confinado a $2 \mathrm{mbar}$ (Termopar a $550^{\circ} \mathrm{C}$ ), $20 \mathrm{~min}$ para o disco paralelo confinado a 4 mbar (Termopar a $550^{\circ} \mathrm{C}$ ) e 27 min para o disco paralelo confinado em cátodo oco a 2 mbar (Termopar a $400^{\circ} \mathrm{C}$ ). Isto possibilitou a determinação da potência térmica na transferência de energia para os discos conforme Tabela 1.

Tabela 1: Análise quantitativa do aço AISI M35 para os revenimentos a plasma.

\begin{tabular}{|c|c|c|c|} 
& \multicolumn{3}{c}{ Condição de revenimento } \\
\cline { 2 - 4 } & $\mathbf{2 ~ \mathbf { ~ m b a r } , \mathbf { 5 5 0 } 0 ^ { \circ } \mathbf { C }}$ & $\mathbf{4} \mathbf{m b a r}, \mathbf{5 5 0}$ & $\mathbf{2} \mathbf{~ m b a r}, \mathbf{4 0 0}{ }^{\circ} \mathbf{C}$ \\
\hline$\Delta \mathrm{E}_{\mathrm{AR}}(\mathrm{J})$ & 1637 & 1152 & 1834 \\
\hline Potência $(\mathrm{W})$ & 0,36 & 0,96 & 1,13 \\
\hline
\end{tabular}

Aplicando-se o mesmo procedimento anterior para os demais tratamentos, ou seja, nos discos revenidos em forno resistivo, cuja espessura em cada condição foi aproximadamente de $0,8 \mathrm{~mm}$, obteve-se os valores da energia transferida para cada tratamento conforme Tabela 2.

Tabela 2: Análise quantitativa do aço AISI M35 para os revenimentos em forno resistivo

\begin{tabular}{c|c|c|c|c|c|c} 
& \multicolumn{6}{|c}{ Temperatura $\left({ }^{\circ} \mathrm{C}\right)$} \\
\cline { 2 - 8 } & 550 & 580 & 610 & 640 & 700 & 750 \\
\hline$\Delta \mathrm{E}_{\mathrm{AR}}(\mathrm{J})$ & 1427 & 1495 & 1597 & 1665 & 1834 & 1970 \\
\hline
\end{tabular}

Os valores das transferências de energia para os discos paralelos tratados sob plasma com diferentes pressões e configurações geométricas de cátodo são mostrados na Fig. 6.

A Fig. 6 indica que a configuração que resultou numa maior transferência de energia durante o tratamento e maiores gradientes térmicos de acordo com as Figs. 4 e 5 respectivamente, foram o disco paralelo confinado a 2 mbar (Termopar a $400^{\circ} \mathrm{C}$ ) e o disco paralelo confinado a 2 mbar (Termopar a $550^{\circ} \mathrm{C}$ ). Isso significa para o caso do disco paralelo confinado a 2 mbar (Termopar a $400^{\circ} \mathrm{C}$ ), que o seu potencial elétrico estava superior ao cátodo, o que aumenta a taxa de colisão dos íons para sua superfície, implicando numa maior temperatura $\left(758^{\circ} \mathrm{C}\right)$, comparado à superfície da amostra que estava abaixo. O mesmo fenômeno ocorreu similarmente para o disco paralelo confinado a 2 mbar (Termopar a $550^{\circ} \mathrm{C}$ ), implicando numa maior temperatura na superfície do disco exposta ao plasma de alta densidade de íons (lado interno confinado do cátodo oco) que foi de $742,35^{\circ} \mathrm{C}$, que a superfície da amostra que estava abaixo.

A configuração cátodo oco sob uma pressão de 4 mbar (Termopar a $550^{\circ} \mathrm{C}$ ), apresentou uma transferência de energia menos intensa para o interior do disco , implicando numa temperatura de $723^{\circ} \mathrm{C}$ na sua superfície, e seus gradientes térmicos terem variação máxima menores de aproximadamente $56^{\circ} \mathrm{C}$, comparado aos valores das amostras tratadas nas configurações cátodo oco a 2 mbar que tiveram variações de temperatura da ordem de $120^{\circ} \mathrm{C}$.

Galvão ${ }^{10}$, também desenvolveu um trabalho que objetivava a determinação do perfil térmico de forma indireta. No seu trabalho, utilizou a configuração cátodo oco cilíndrico, em discos de $3 \mathrm{~mm}$ de espessura e $12 \mathrm{~mm}$ de diâmetro, e obteve gradiente térmico máximo em torno de $130^{\circ} \mathrm{C}$ em seu interior, no tratamento a pressão de 4 mbar, conforme Fig. 7. Os resultados diferem consideravelmente daqueles apresentados neste trabalho. Esta diferença é creditada às diferenças geométricas das amostras que neste trabalho optou-se por uma geometria que aproximasse o fluxo térmico para uma situação unidimensional

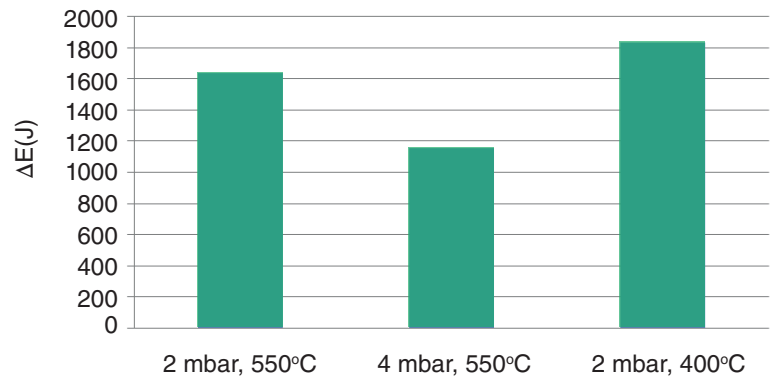

Figura 6: Gráfico mostrando os valores das transferências de energia obtidas dos discos paralelos tratados termicamente por plasma.

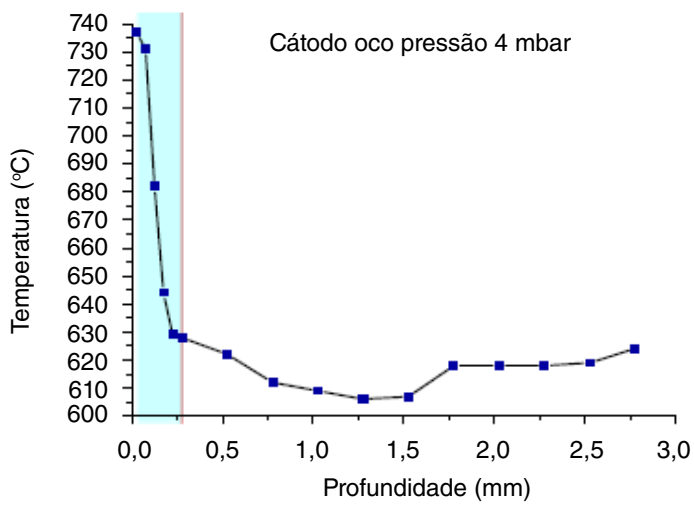

Figuras 7: Tratamento de revenimento realizado sob plasma por na configuração cátodo oco cilíndrico sob uma pressão de 4 mbar.

\section{CONCLUSÕES}

Um método para medir temperaturas pontuais de sólidos imersos em plasma foi apresentado. Através do método foi possível determinar o perfil térmico de discos de aço AISI M35 exposto simultaneamente a duas configurações de plasma. Enquanto uma das superfícies estava exposta a uma descarga de cátodo oco, a outra superfície estava exposta a uma descarga planar. Os valores de microdureza nas amostras revenidas em plasma variaram consideravelmente 
entre a região exposta ao plasma de alta densidade de íons (cátodo oco) e a superfície exposta ao plasma de menor densidade (plasma planar). Como consequência, diferenças de temperaturas tão altas quanto $120^{\circ} \mathrm{C}$ foi determinada entre a superfície exposta à descarga de cátodo oco e o interior do disco. Foi verificado também que as superfícies expostas ao plasma planar aqueceram menos que as superfície expostas ao plasma de cátodo oco.

\section{AGRADECIMENTOS}

Os autores agradecem à CAPES pelo apoio financeiro necessário para realizar este trabalho.

\section{REFERÊNCIAS}

1. YAMAZAKI, K. et al. PAS (Plasma activated sintering): Transient sintering process control for rapid consolidation of powders. Journal of Materials Processing Technology, v. 56, n. 1-4, p. 955-965. 1996.

2. KERSTEN, $\mathrm{H}$. et al. The energy balance at substrate surfaces during plasma processing. Vacuum, vol. 63, n. 3, p. 385-431, 2001.
3. AKAMATSU, $H_{\text {.; }}$ YATSUZUKA, M. Simulation of surface temperature of metals irradiated by intense pulsed electron, ion and laser beams. Surface and Coatings Technology, v. 169-170, n. 0, pp. 219-222, 2003.

4. ALVES JR, C.; HAJEK, V.; SANTOS, C. A. Thermal behavior of supersolidus bronze powder compacts during heating by hollow cathode discharge. Materials Science and Engineering, vol. 348, n.1, pp. 84-89 ( 2003)

5. GALVÃO, N. K. A. M. et al. Structural modifications of M35 stee submitted to thermal gradients in plasma reactor. Journal of Materials Processing Technology, v. 200, n. 1-3, p. 115-119, 2008

6. BARANKOVA, H.; BARDOS, L.; BERG, S. The radio frequency hollow cathode plasma jet arc for the film deposition. Journal of Vacuum Science \& Technology a-Vacuum Surfaces and Films, vol. 14, n. 6, p. 3033-3038, Nov-Dec, 1996.

7. INCROPERA, F. P.; DEWITT, D. P. Fundamentos de Transferência de Calor e de Massa. $5^{\circ}$ edição. 2003.

8. GUIDORIZZI, H., L. Um curso de cálculo, volume $1,5^{\circ}$ edição, Rio de Janeiro. 2008

9. GALVÃO. Determinação do perfil térmico em amostras de aço AISI M35 imersas em plasma. 2007. Dissertação de mestrado. programa de pós-graduação em ciência e engenharia de materiais, UFRN. 